\title{
The effect of fractal-like mechanical ventilation on vital signs in a rat model of acute-on-chronic liver failure
}

\author{
Arman Nataj ${ }^{1 a}$, Golnar Eftekhariia, Mohammad R. Raoufy ${ }^{1}$, Ali R. Mani ${ }^{2 *}$ \\ ${ }^{1}$ Department of Physiology, Faculty of Medical Sciences, Tarbiat Modares University, Tehran, Iran \\ ${ }^{2}$ Network Physiology Lab, Division of Medicine, University College London, London, UK \\ ${ }^{a}$ These authors are joint first authors \\ *Corresponding Authors: \\ Alireza Mani MD, PhD \\ Institute of Liver and Digestive Health \\ Division of Medicine \\ Royal Free Campus \\ University College London (UCL) \\ Rowland Hill Street, London, NW3 2PF, UK \\ Emails: a.r.mani@ucl.ac.uk, Tel/Fax:+44(0)2074332878
}

Electronic Word Count: 3972

Conflict of interest: The authors declare that the research was conducted in the absence of any commercial or financial relationships that could be construed as a potential conflict of interest 


\begin{abstract}
The network of interactions between different organs is impaired in liver cirrhosis. Liver cirrhosis is associated with multi-system involvement which eventually leads to multiple organ failure. This process is accelerated by a precipitating factor such as bacterial infection which leads to respiratory distress, circulatory shock, neural dysfunction and very high mortality. Cirrhotic patients often have blunted respiratory sinus arrhythmia and impaired cardio-respiratory variability. Fractal-like mechanical ventilation is reported to enhance respiratory sinus arrhythmia and attenuate respiratory distress in experimental models. In present study we hypothesised that fractal-like mechanical ventilation may improve the outcome of cirrhotic rats with multiple organ failure. Cirrhosis was induced by chronic biliary obstruction in rats. Acute multiple organ failure was induced by intraperitoneal injection of bacterial endotoxin in cirrhotic rats. The effect of conventional mechanical ventilation (with constant tidal volume and respiratory rate) or fractal-like ventilation (with the same average but variable tidal volume and respiratory rate) were assessed on vital signs, oxygen saturation and plasma alanine aminotransferase in anaesthetised cirrhotic rats. We demonstrated that fractal-like mechanical ventilation was accompanied by improved oxygen saturation, reduced heart rate and decreased liver injury following injection of bacterial endotoxin. Moreover, variable mechanical ventilation in cirrhotic rats reduced mortality and prevented a fall in short-term heart rate variability following endotoxin challenge in comparison with rats with constant mechanical ventilation. We suggest further investigations on beneficial effects of fractal-like ventilation strategy in critically ill patients with liver failure requiring organ support and mechanical ventilation.
\end{abstract}

Key words: Cirrhosis, Endotoxin, Fractal, Liver failure, Mechanical ventilation, Network physiology 


\section{Introduction}

Liver cirrhosis is a major cause of mortality worldwide with a rate of $\sim 2$ million deaths per year (Byass 2014). In addition to specific liver-related complications, cirrhosis is associated with multisystem involvement which leads to impaired cardiovascular, respiratory, renal, neural and immune function. The process of multiple organ failure in cirrhosis can be accelerated by a precipitating factor such as bacterial infection (Jalan et al 2012). In fact, bacterial infection results in acute-on-chronic liver dysfunction and accelerated failure of other organs (Mücke et al 2017). Cirrhotic patients with multiple organ failure often have poor prognosis and require intensive care unit admission (O 'brien et al 2012).

The complex interaction between different organs system in cirrhosis makes it an interesting topic for investigation within the context of Network Physiology. The mechanism of progressive organs failure is not well understood in patients with acute-on-chronic liver failure. It appears that the network of interactions between different organs is impaired in cirrhosis. For examples, the interaction between respiratory cycles and heart rhythm is blunted in cirrhosis (i.e. reduced respiratory sinus arrhythmia). Respiratory sinus arrhythmia is crucial for optimum tissue oxygenation and cardiac function (Ben-Tal et al 2012). Patients with cirrhosis often have reduced heart rate variability (HRV) which carries a poor prognosis in this patient population (Mani et al 2009, Bhogal et al 2018). Respiratory cycles in healthy individuals is associated with fractal-like fluctuations in rate and volume (Raoufy et al 2016). Although such complex fluctuations enhances tissue oxygenation (Mutch et al 2005, Raoufy et al 2017), most conventional mechanical ventilators are designed to deliver constant rhythm and do not mimic physiological breath-to-breath fractal-like variations in the respiratory rate and volume. Mutch et al., reported that mechanical ventilation of anesthetised pigs with a fractal-like rhythm improves respiratory sinus arrhythmia (Mutch et al 2005). Other reports indicate that the outcome in animals model of acute respiratory distress syndrome and asthma is improved following variable mechanical ventilation (llka et al 2018, Boker et al 2002).

The outcome of cirrhotic patients requiring mechanical ventilation in the intensive care unit is very poor and still no specific therapy is available to improve their survival (Levesque et al 2014). We hypothesise that variable mechanical ventilation may improve the outcome in such critically ill patients. This study was aimed to test this hypothesis in a rat model of acute-on-chronic liver failure.

\section{Method}

2.1. Ethics statement: All animal procedures were approved by the Ethics committee of Tarbiat Modares University. All animal procedures were in accordance with recommendations of $\mathrm{NIH}$ guidelines on animal experimentation (publication no. 85-23). 6-8 rats were used in each experimental group.

2.2. Animal model of acute-on-chronic liver failure: Liver cirrhosis was induced by surgical ligation of bile duct under general anaesthesia as described (Mani et al 2006). Bile duct ligated (BDL) and sham-operated (SHAM) control rats had access to food and water ad libitum and were used for the next step of experiments four weeks after the operation. Injection of bacterial lipopolysaccharide (LPS) in cirrhotic rats induces rapid deterioration of liver function and precipitates multiple organ failure (Wright et al 2007, Harry et al 1999). Thus, acute-on-chronic liver failure was induced by intraperitoneal injection of a single dose of LPS $(1 \mathrm{mg} / \mathrm{kg}$, extracted from Salmonella typhimurium, Sigma, UK) (Haddadian et al 2013).

2.3. Experimental design and mechanical ventilation: Four weeks after bile duct ligation or sham surgery, animals were mechanically ventilated under general anaesthesia as described (Ilka et al 
2018). In brief, the trachea was cannulated and connected to a volume controlled ventilator (Harvard Apparatus, Holliston, MA). Animals were randomly assigned to have either constant or fractal-like mechanical ventilation. In the constant ventilation group, a non-variable tidal volume $(1.7 \mathrm{ml})$ and respiratory rate $(77 \mathrm{breath} / \mathrm{min})$ were given with a positive end-expiratory pressure (PEEP) of $5 \mathrm{~cm} \mathrm{H}_{2} \mathrm{O}$. The fractal-like ventilation groups received mechanical ventilation with the same average but a variable tidal volume and respiratory rate to obtain a minute ventilation equal to that during constant ventilation (Ilka et al 2018). PEEP was kept at $5 \mathrm{~cm} \mathrm{H}_{2} \mathrm{O}$ in both groups. We used an application programming interface which communicates with the ventilator through a RS232C serial interface. This interface allows to control the rate and volume of each cycle of mechanical ventilation directly from the MATLAB command line. 1000 cycles of respiratory rate and tidal volume obtained from the pattern of physiological healthy breathing were continuously looped itself until the end of the experiments (Ilka et al 2018). In fractal-like ventilation group: animals were ventilated with a variable tidal volume (mean $=1.7 \mathrm{ml}$, coefficient of variation [CV]=7.4\%) and respiratory rate (mean=77 breaths/min, $\mathrm{CV}=7.1 \%$ ). Rats were placed on a heated pad to maintain their body temperature throughout mechanical ventilation. Fractal-like behaviour of inter-breath interval variations were assessed using detrended fluctuation analysis (DFA) (Peng et al 1995, Ma Qianli D Y, Bartsch et al 2010, Chen et al 2005, 2002). ECG, systolic blood pressure and oxygen saturation ( $\left.\mathrm{SpO}_{2}\right)$ were recorded using a Powerlab system (ADInstrument, Australia). One hour after the commence of mechanical ventilation, LPS (1 mg/kg) or saline were injected intraperitoneally and animals were followed for 4 hours. In a separate group of animals, blood samples were taken 2 hours after LPS or saline injection for measurement of plasma alanine aminotransferase (ALT) using a routine biochemical assay.

2.4. Heart rate variability analysis: $E C G$ signals were recorded at the sampling rate of $10 \mathrm{kHz}$ using a Powerlab system. The $\mathrm{R}$ peaks were detected and the $\mathrm{R}-\mathrm{R}$ interval series was generated using an $\mathrm{ad}$ hoc computer program. The standard deviation of the R-R intervals (SDNN) was calculated as a measure of total HRV in 5 min ECG recordings. Short-term and long-term HRV were measured by calculating SD1 and SD2 using the Poincare' plot respectively (Haddadian et al 2013).

2.5. Statistical analysis: Data are expressed as mean \pm SEM. Two-way analysis of variance was used for statistical analysis followed by post-hoc Tukey's test. $\mathrm{P}<0.05$ was considered statistically significant.

\section{Results}

BDL rats showed clinical manifestations biliary cirrhosis such as development of ascites, jaundice and elevation of plasma liver enzymes (e.g. ALT). Post-mortem analysis also showed a significant increase in spleen weight (in index of portal hypertension) as well as liver stiffness (data not shown). Detrended fluctuation analysis of inter-breath intervals in group of rats with variable mechanical ventilation demonstrates a linear correlation between the $\log$ (scale) and $\log$ (fluctuations) as shown in figure 1. This indicated that variable mechanical ventilation has a fractal-like dynamic similar to healthy individuals (Raoufy et al 2016). Similar fractal-like pattern of fluctuation was observed in tidal volume fluctuations in the variable mechanical ventilation group as expected (data not shown).

Figure 2 shows plasma level of ALT in four experimental groups. Two-way ANOVA showed a significant interaction between type of mechanical ventilation and type of surgery (SHAM versus $B D L)(P=0.0061)$. This indicates that fractal-like ventilation has different effect on plasma liver enzyme level in SHAM versus BDL group. There was a statistically significant difference in plasma ALT 
levels between BDL rats which had constant or fractal-like mechanical ventilation $(P<0.001$, Tukey's post-test).

The effect of fractal-like mechanical ventilation on heart rate, systolic blood pressure and $\mathrm{SpO}_{2}$ is demonstrated in figure 3 . In general, BDL rats exhibited lower heart rate and systolic blood pressure in comparison with SHAM groups, a phenomenon which goes along with previous reports (Mani et al 2006, Gaskari et al 2015, 2002). BDL-fractal group showed even lower heart rate and blood pressure in comparison with $\mathrm{BDL}$ rats with constant ventilation. $\mathrm{BDL}$ rats with constant mechanical ventilation exhibited a steep drop in oxygen saturation $\left(\mathrm{SpO}_{2}\right) 3$ hours post LPS injection which was statistically significant compared to SHAM rats as well as BDL rats given fractal-like mechanical ventilation (figure 3C).

The effect of fractal-like ventilation on HRV indexes is shown in figure 4. We did not observe a significant difference in SDNN between experimental groups. Poincare' plot was used to compute short-term and long-term variability separately. Short-term HRV (SD1) dropped significantly in BDL rats with constant mechanical ventilation after endotoxin challenge in comparison with BDL-fractal group as well as SHAM rats. There was no significant difference in long-term HRV (SD2) between the experimental groups.

The effect of variable mechanical ventilation on survival is depicted in figure 5 . Mantel-Cox test indicated a significant difference in survival between the groups (Chi-square $=6.26, P=0.036$ ). Most SHAM rats survived the endotoxin challenge within 4 hours. There was high mortality in BDL group within 4 hours of LPS injection despite mechanical ventilation. Four-hour survival rate was higher in $\mathrm{BDL}$ rats given fractal-like rather than constant mechanical ventilation.

\section{Discussion}

Chronic liver failure is a multisystem disease and eventually leads to multiple organ failure, a process with is often accelerated by bacterial infection/sepsis. Cirrhotic patients with sepsis are often admitted to intensive care units and may require mechanical ventilation (Gustot et al 2009). However, the survival rate of cirrhotic patients requiring mechanical ventilation is lower than patients without underlying liver disease (Rabe et al 2004). Although the mechanism of endotoxininduced acute respiratory distress syndrome has been studied in cirrhotic rats in the past (Chang and Ohara 1994), still no specific therapy is available. In present study we hypothesised that fractal-like mechanical ventilation may improve the outcome in such critically ill patients and tested this hypothesis in a rat model of acute-on-chronic liver failure. Our results showed that fractal-like ventilation in cirrhotic rats was associated with less elevation of plasma transaminase (ALT) which indicates less severity of acute-on-chronic liver injury following LPS injection in the fractal-like group. In addition, we did not observe a steep drop in $\mathrm{SpO}_{2}$ in cirrhotic rats with fractal-like mechanical ventilation in comparison with cirrhotic rats with constant mechanical ventilation. This observation goes along with less severity of respiratory distress and corroborates with previous reports showing that acute respiratory distress syndrome is attenuated following fractal-like mechanical ventilation in a pig model (Boker et al 2002).

Although these results are promising, the exact mechanism is not investigated in the present study. However, one may speculate that variable mechanical ventilation my directly affect the airways (Ilka et al 2018) or indirectly affect the autonomic control system through neural feedback mechanisms (Mutch et al 2005). Mutch and colleagues reported that fractal ventilation enhances respiratory sinus arrhythmia (Mutch et al 2005). It is well known that vagus nerve is an important component of 
respiratory sinus arrhythmia (Katona and Jih 1975). The vagus nerve also plays a pivotal role in prevention of organ failure during systemic inflammation (Tracey 2007, Rosas-Ballina et al 2011). Tracey and colleagues, demonstrated the existence of a neuronal circuit termed "the cholinergic anti-inflammatory pathway" in which the major component is the descending branch of the vagus nerve (Tracey 2007). Based on this model, action potentials coming from the vagus nerve inhibit the production of pro-inflammatory cytokines through activation of alpha7 nicotinic acetylcholine receptors (Tracey 2007, Mazloom et al 2013). The mechanism of such immune modulation is not precisely known, but experimental evidence suggests that vagus nerve stimulation leads to activation of a subset of T lymphocytes that express choline acetyltransferase (Rosas-Ballina et al 2011). These T cells migrate to the site of inflammation and release acetylcholine that inhibits macrophage function (Rosas-Ballina et al 2011). The discovery of this neural anti-inflammatory pathway is important within the context of cirrhosis for two reasons: Firstly, this model explains why there is an inverse correlation between plasma inflammatory cytokines (e.g. interleukin-6) and indexes of vagal activity in patients with cirrhosis (Mani et al 2009). Secondly, it introduces the possibility of vagus nerve stimulation as a potential therapy for management of inflammatory conditions such as acute-on-chronic liver failure where an exaggerated immune response may cause harm (Eftekhari et al 2014, Hajiasgharzadeh and Baradaran 2017). The effect of fractal-like ventilation in rats with acute-on-chronic liver disease is in line with the latter. Our results showed that fractal-like ventilation decreases heart rate and blood pressure and prevent a drop in shortterm HRV (an index of respiratory sinus arrhythmia). We have previously shown that liver cells express alpha-7 nicotinic acetylcholine receptors (Hajiasgharzadeh et al 2014) and central neural mechanisms can modulate the severity of liver injury (Eftekhari et al 2014). Therefore, our observations corroborate with the effect of fractal ventilation on modulation of vagus nerve activity, although further studies are required to prove this explanation.

We demonstrated that fractal-like mechanical ventilation is accompanied by a decrease in shortterm mortality, improved oxygen saturation and decreased liver injury following injection of bacterial endotoxin. Future investigation can pave the way to understand the mechanism of these effects by using an electrophysiologic (direct vagus nerve recording) or pharmacologic approach. Development of new analytical methods in Network Physiology will also help to uncover the mechanism of multiple organ failure in cirrhosis form a different perspective (Bartsch et al 2015, Xiong et al 2017, Asada et al 2016, Ivanov et al 2016, Kanter et al 2015). For example, multiscale network construction (Shashikumar et al 2017) can be employed for quantification of cardiovascular and respiratory interaction within the context of sepsis in patients with liver failure. Such novel approaches are crucial as classical medicine currently lacks a good pathophysiological model to explain the mechanism of multiple organ failure in acute-on-chronic liver failure.

In the present study we used analytical techniques that were previously developed for analysis of physiological fluctuations in health and disease. Our aim was to introduce a method to ameliorate acute-on-chronic liver failure by alteration of respiratory pattern in an animal model of multiorgan failure. The association between respiratory pattern and liver function has not been studied previously and a Network approach can potentially explain the complex interaction between multiple organs during systemic inflammation. The major limitation of our study is that we have not developed a novel computational tool to assess interaction between different organs in liver failure. However, our study introduces a new phenomenon which is suitable for analysis using techniques that are being developed in the emerging field of Network Physiology (Bashan et al 2012, Bartsch et al 2015, Ivanov et al 2016, Kanter et al 2015). In recent past decades hepatology has benefited enormously from collaboration with other scientific communities (Shirazi et al 2016). A multidisciplinary approach is more likely to introduce alternative models and uncover the mechanism of experimental therapies such as fractal-like ventilation in patients with liver failure. 
Acknowledgment: The authors declare no conflict of interest. This work was supported by grants from Tarbiat Modares University.

\section{References}

Asada T, Aoki Y, Sugiyama T, Yamamoto M, Ishii T, Kitsuta Y, Nakajima S, Yahagi N and Doi K 2016 Organ System Network Disruption in Nonsurvivors of Critically III Patients. Crit. Care Med. 44 83-90 Online: https://insights.ovid.com/crossref?an=00003246-201601000-00010

Bartsch R P, Liu K K L, Bashan A and Ivanov P C 2015 Network Physiology: How Organ Systems Dynamically Interact ed M Perc PLoS One 10 e0142143 Online: http://dx.plos.org/10.1371/journal.pone.0142143

Bashan A, Bartsch R P, Kantelhardt J W, Havlin S and Ivanov P C 2012 Network physiology reveals relations between network topology and physiological function Nat. Commun. 3 Online: www.nature.com/naturecommunications

Ben-Tal A, Shamailov S S and Paton J F R 2012 Evaluating the physiological significance of respiratory sinus arrhythmia: Looking beyond ventilation-perfusion efficiency J. Physiol. 590 1989-2008

Bhogal A S, De Rui M, Pavanello D, El-Azizi I, Rowshan S, Amodio P, Montagnese S and Mani A R 2018 Which heart rate variability index is an independent predictor of mortality in cirrhosis Dig. Liver Dis. Online: https://doi.org/10.1016/j.dld.2018.09.011

Boker A, Graham M R, Walley K R, Manus B M, Girling L G, Walker E, Lefevre G and Mutch W A 2002 Improved arterial oxygenation with biologically variable or fractal ventilation using low tidal volumes in a porcine model of acute respiratory distress syndrome. Am J Respir Crit Care Med 165 456-62

Byass P 2014 The global burden of liver disease: A challenge for methods and for public health $B M C$ Med. 12 Online: http://www.biomedcentral.com/1741-7015/12/145/abstract.

Chang S W and Ohara N 1994 Chronic biliary obstruction induces pulmonary intravascular phagocytosis and endotoxin sensitivity in rats. J. Clin. Invest. 94 2009-19 Online: http://www.ncbi.nlm.nih.gov/pubmed/7962547

Chen Z, Hu K, Carpena P, Bernaola-Galvan P, Stanley H E and Ivanov P C 2005 Effect of nonlinear filters on detrended fluctuation analysis Phys. Rev. E 71011104 Online: http://www.ncbi.nlm.nih.gov/pubmed/15697577

Chen Z, Ivanov P C, Hu K and Stanley H E 2002 Effects of nonstationarities on detrended fluctuation analysis Phys. Rev. E 65 Online: http://cds.cern.ch/record/526487/files/0111103.pdf

Eftekhari G, Hajiasgharzadeh K, Ahmadi-Soleimani S M, Dehpour A R, Semnanian S and Mani A R 2014 Activation of central muscarinic receptor type 1 prevents development of endotoxin tolerance in rat liver Eur. J. Pharmacol. $\mathbf{7 4 0}$

Gaskari S A, Liu H, Mello C D', Kunos G and Lee S S 2015 Blunted cardiac response to hemorrhage in cirrhotic rats is mediated by local macrophage-released endocannabinoids J. Hepatol. 62 12727 Online: https://www.journal-of-hepatology.eu/article/S0168-8278(15)00052-5/pdf

Gaskari S A, Mani A R, Ejtemaei-Mehr S, Namiranian K, Homayoun H, Ahmadi H and Dehpour A R 2002 Do endogenous opioids contribute to the bradycardia of rats with obstructive cholestasis? Fundam. Clin. Pharmacol. 16

Gustot T, Durand F, Lebrec D, Vincent J-L and Moreau R 2009 Severe sepsis in cirrhosis Hepatology 50 2022-33 Online: http://doi.wiley.com/10.1002/hep.23264

Haddadian Z, Eftekhari G, Mazloom R, Jazaeri F, Dehpour A R and Mani A R 2013 Effect of endotoxin 
on heart rate dynamics in rats with cirrhosis Auton. Neurosci. Basic Clin. 177

Hajiasgharzadeh K and Baradaran B 2017 Cholinergic anti-inflammatory pathway and the liver Adv. Pharm. Bull. 7 507-13 Online: http://apb.tbzmed.ac.ir

Hajiasgharzadeh K, Tavangar S M, Javan M, Dehpour A R and Mani A R 2014 Does hepatic vagus nerve modulate the progression of biliary fibrosis in rats? Auton. Neurosci. Basic Clin. 185

Harry D, Anand R, Holt S, Davies S, Marley R, Fernando B, Goodier D and Moore K 1999 Increased sensitivity to endotoxemia in the bile duct-ligated cirrhotic rat Hepatology $301198-205$

Ilka F, Javan M and Raoufy M R 2018 Variable ventilation decreases airway responsiveness and improves ventilation efficiency in a rat model of asthma Respir. Physiol. Neurobiol. 255 39-42 Online: https://doi.org/10.1016/j.resp.2018.05.007

Ivanov P C, Liu K K L and Bartsch R P 2016 Focus on the emerging new fields of network physiology and network medicine New J. Phys. 18100201 Online: http://stacks.iop.org/13672630/18/i=10/a=100201?key=crossref.4bed6cebdb07e8448bd88090bce6a85a

Jalan R, Gines P, Olson J C, Mookerjee R P, Moreau R, Garcia-Tsao G, Arroyo V and Kamath P S 2012 Acute-on chronic liver failure J. Hepatol. 57 1336-48 Online: https://ac.elscdn.com/S0168827812005193/1-s2.0-S0168827812005193-main.pdf?_tid=11f22717-a96647e8-a82e-851b863be07a\&acdnat=1530633495_aabf60c779aaa82198d574b282c4d62e

Kanter I, Stramaglia S, degli Studi di Bari U, Wolfgang Kinzel I, Ch Ivanov P, L Liu K K, Bartsch R P, Lin A and Mantegna R N 2015 Plasticity of brain wave network interactions and evolution across physiologic states Plasticity of brain wave network interactions Front. Neural Circuits / www.frontiersin.org 962 Online: www.frontiersin.org

Katona P G and Jih F 1975 Respiratory sinus arrhythmia: noninvasive measure of parasympathetic cardiac control JOURNALOF Appl. Physiol. 39 Online: www.physiology.org/journal/jappl

Levesque E, Saliba F, Ichaï P and Samuel D 2014 Outcome of patients with cirrhosis requiring mechanical ventilation in ICU J. Hepatol. 60 570-8 Online: https://ac.elscdn.com/S0168827813008131/1-s2.0-S0168827813008131-main.pdf?_tid=147e0ae6-596e47b5-8045-4311c7c4bfb2\&acdnat=1530632352_b0dc6795fe310fca8c82b39313d14fa3

Ma Qianli D Y, Bartsch R P, Yoneyama, Bernaola-galv P and Ivanov P C 2010 Effect of extreme data loss on detrended fluctuation analysis Phys Rev E Stat Nonlin Soft Matter Phys 81 1-16 Online: https://www.ncbi.nlm.nih.gov/pmc/articles/PMC3534784/pdf/nihms424658.pdf

Mani A R, Ippolito S, Ollosson R and Moore K P 2006 Nitration of cardiac proteins is associated with abnormal cardiac chronotropic responses in rats with biliary cirrhosis Hepatology 43

Mani A R, Montagnese S, Jackson C D, Jenkins C W, Head I M, Stephens R C, Moore K P and Morgan M Y 2009 Decreased heart rate variability in patients with cirrhosis relates to the presence and degree of hepatic encephalopathy Am. J. Physiol. - Gastrointest. Liver Physiol. 296

Mazloom R, Eftekhari G, Rahimi M, Khori V, Hajizadeh S, Dehpour A R and Mani A R 2013 The role of $\alpha 7$ nicotinic acetylcholine receptor in modulation of heart rate dynamics in endotoxemic rats PLoS One 8

Mücke M M, Rumyantseva T, Mücke V T, Schwarzkopf K, Joshi S, Kempf V A J, Welsch C, Zeuzem S and Lange C M 2017 Bacterial infection-triggered acute-on-chronic liver failure is associated with increased mortality Liver Int. 12 3218-21 Online: http://doi.wiley.com/10.1111/liv.13568

Mutch W A C, Graham M R, Girling L G and Brewster J F 2005 Fractal ventilation enhances respiratory sinus arrhythmia Respir. Res. 6 Online: https://www.ncbi.nlm.nih.gov/pmc/articles/PMC1142342/pdf/1465-9921-6-41.pdf

O 'brien A J, Welch C A, Singer M, Harrison D A, O 'brien A J, Singer Á M, Welch C A and Harrison D A 2012 Prevalence and outcome of cirrhosis patients admitted to UK intensive care: a 
comparison against dialysis-dependent chronic renal failure patients Online:

https://link.springer.com/content/pdf/10.1007\%2Fs00134-012-2523-2.pdf

Peng C -K., Havlin S, Stanley H E and Goldberger A L 1995 Quantification of scaling exponents and crossover phenomena in nonstationary heartbeat time series Chaos An Interdiscip. J. Nonlinear Sci. 5 82-7 Online: http://www.ncbi.nlm.nih.gov/pubmed/11538314

Rabe C, Schmitz V, Paashaus M, Musch A, Zickermann H, Dumoulin F-L, Sauerbruch T and Caselmann W 2004 Does intubation really equal death in cirrhotic patients? Factors influencing outcome in patients with liver cirrhosis requiring mechanical ventilation Intensive Care Med. 30 1564-71 Online: http://link.springer.com/10.1007/s00134-004-2346-x

Raoufy M R, Ghafari T, Darooei R, Nazari M, Mahdaviani S A, Eslaminejad A R, Almasnia M, Gharibzadeh S, Mani A R and Hajizadeh S 2016 Classification of asthma based on nonlinear analysis of breathing pattern PLoS One 11

Raoufy M R, Ghafari T and Mani A R 2017 Complexity analysis of respiratory dynamics Am. J. Respir. Crit. Care Med. 196

Rosas-Ballina M, Olofsson PS, Ochani M, Valdés-Ferrer SI, Levine Y A, Reardon C, Tusche M W, Pavlov V A, Andersson U, Chavan S, Mak T W, Tracey K J and Edu K 2011 AcetylcholineSynthesizing T Cells Relay Neural Signals in a Vagus Nerve Circuit Science (80-. ). 334 98-101 Online: www.sciencemag.org/cgi/content/full/science.1209985/DC1

Shashikumar S P, Li Q, Clifford G D and Nemati S 2017 Multiscale network representation of physiological time series for early prediction of sepsis Physiol. Meas. 38 2235-48 Online: http://stacks.iop.org/0967$3334 / 38 / \mathrm{i}=12 / \mathrm{a}=2235$ ?key=crossref.9c3aaceab6c6959fbb8eca0a976755a4

Shirazi A H, Modiri A B, Heydari S, Rohn J L, Jafari G R and Mani A R 2016 Evolution of Communities in the Medical Sciences: Evidence from the Medical Words Network PLoS One $\mathbf{1 1}$

Tracey K J 2007 Physiology and immunology of the cholinergic antiinflammatory pathway. J. Clin. Invest. 117 289-96 Online: http://www.ncbi.nlm.nih.gov/pubmed/17273548

Wright G, Davies N A, Shawcross D L, Hodges S J, Zwingmann C, Brooks H F, Mani A R, Harry D, Stadlbauer V, Zou Z, Williams R, Davies C, Moore K P and Jalan R 2007 Endotoxemia produces coma and brain swelling in bile duct ligated rats Hepatology 45

Xiong W, Faes L and Ch Ivanov P 2017 Entropy measures, entropy estimators, and their performance in quantifying complex dynamics: Effects of artifacts, nonstationarity, and long-range correlations Phys. Rev. E 9562114 Online: https://journals.aps.org/pre/pdf/10.1103/PhysRevE.95.062114 
Figure 1. Detrended fluctuation analysis for inter-breath interval time-series in a representative fractal-like mechanical ventilation group. The scaling exponents $(\alpha=0.8)$ in this graph is within the range of the scaling exponent of respiratory fluctuations in healthy individuals (Raoufy et al 2016).

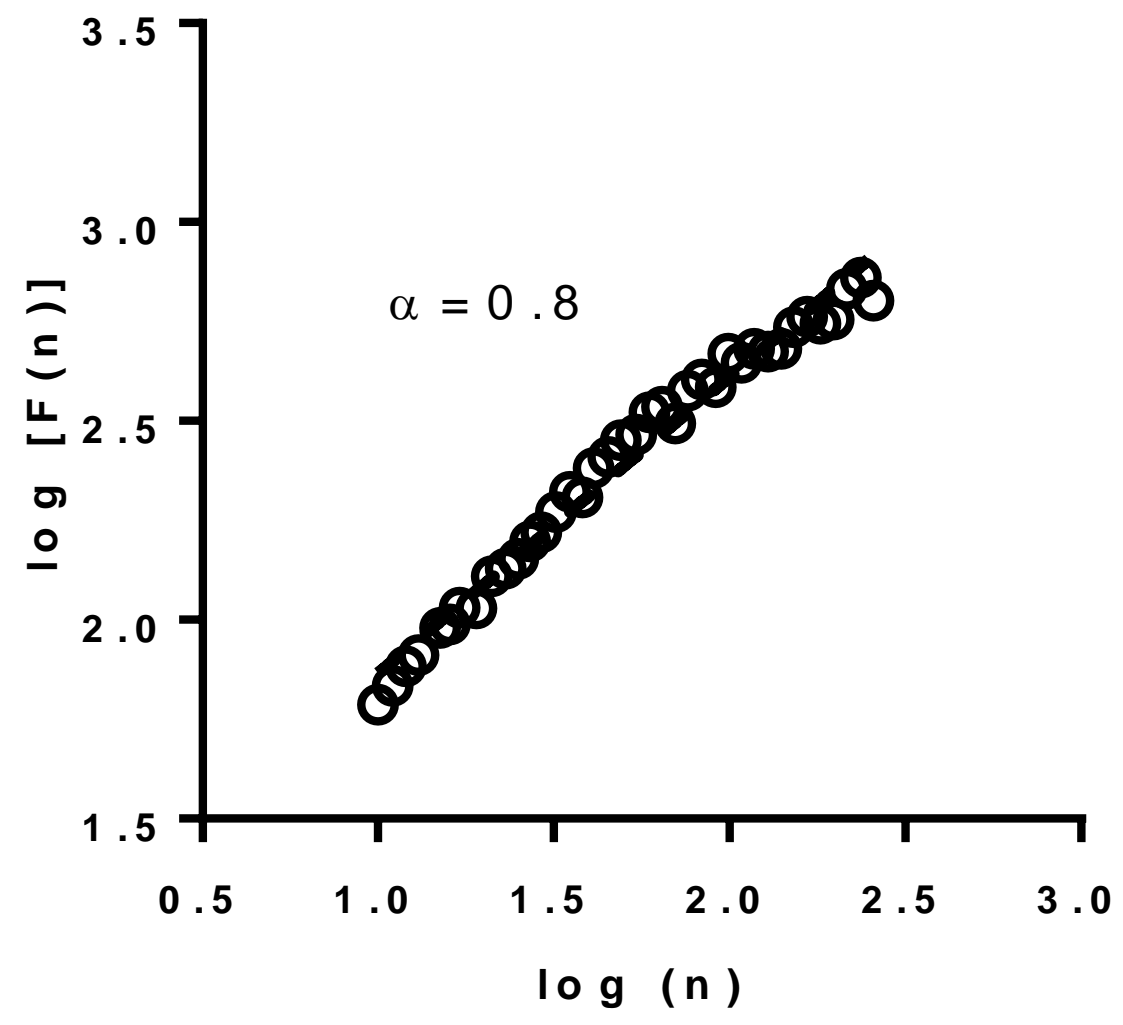


Figure 2. The effect of fractal-like mechanical ventilation on plasma transaminase (ALT) levels in control (SHAM) and cirrhotic (BDL) rats after 2 hours challenge with endotoxin (LPS). Fractal-like breathing in cirrhotic rats was associated with lower ALT levels in comparison with rats given constant ventilation. ${ }^{* * *} \mathrm{P}<0.001$.

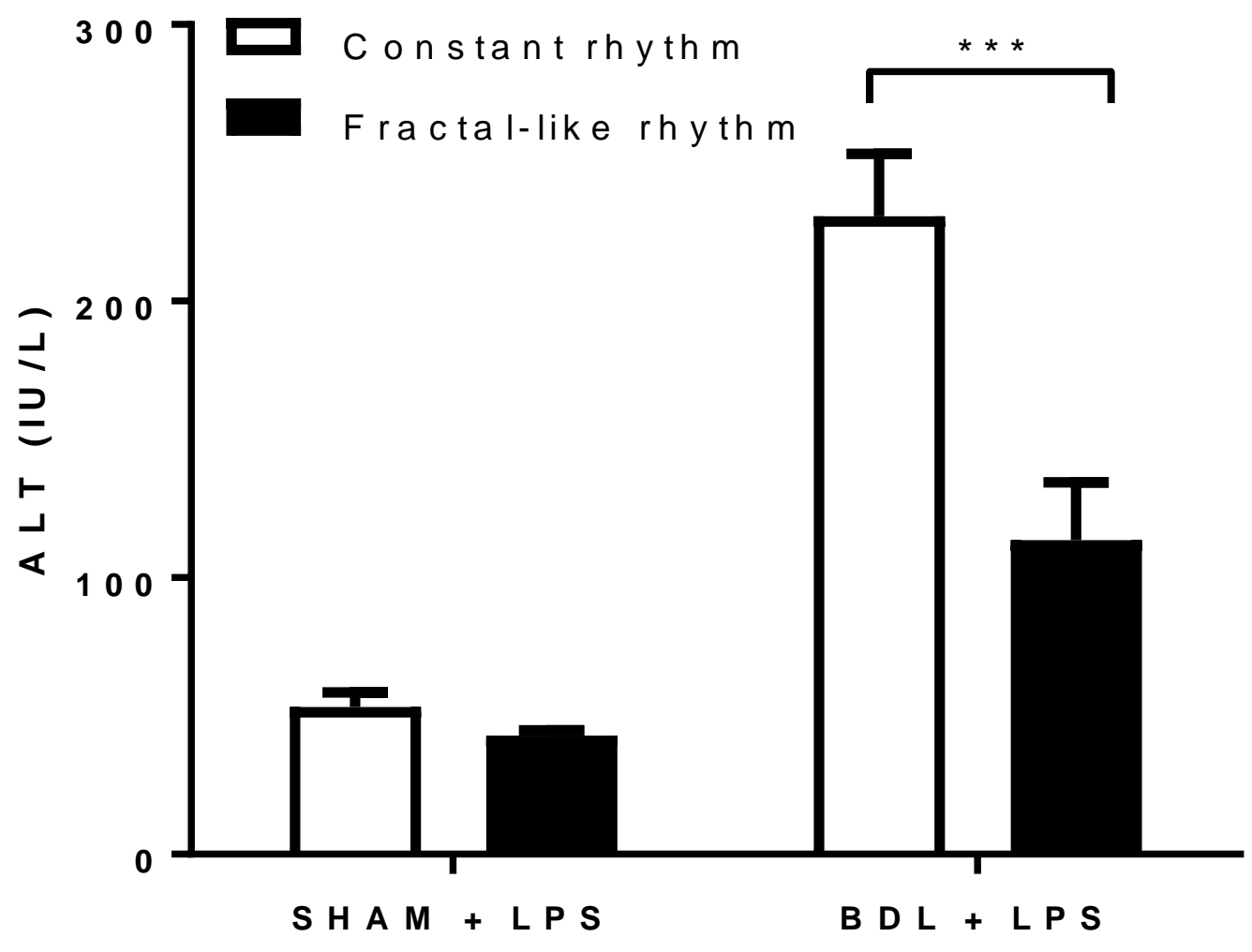


Figure 3. The effect of fractallike mechanical ventilation on mean heart rate $(A)$, systolic blood pressure (B) and $\mathrm{SpO}_{2}$ (C). a: $P<0.0001 \mathrm{BDL}$ fractal vs. BDL constant. b: BDL constant ventilation vs. SHAM constant $\mathrm{P}<0.0001$. $^{*}$ : cirrhotic constant vs. SHAM constant (Tukey's post-test) $\mathrm{P}<0.05$. \#: cirrhotic fractal vs. cirrhotic constant (Tukey's post-test) $\mathrm{P}<0.05$.

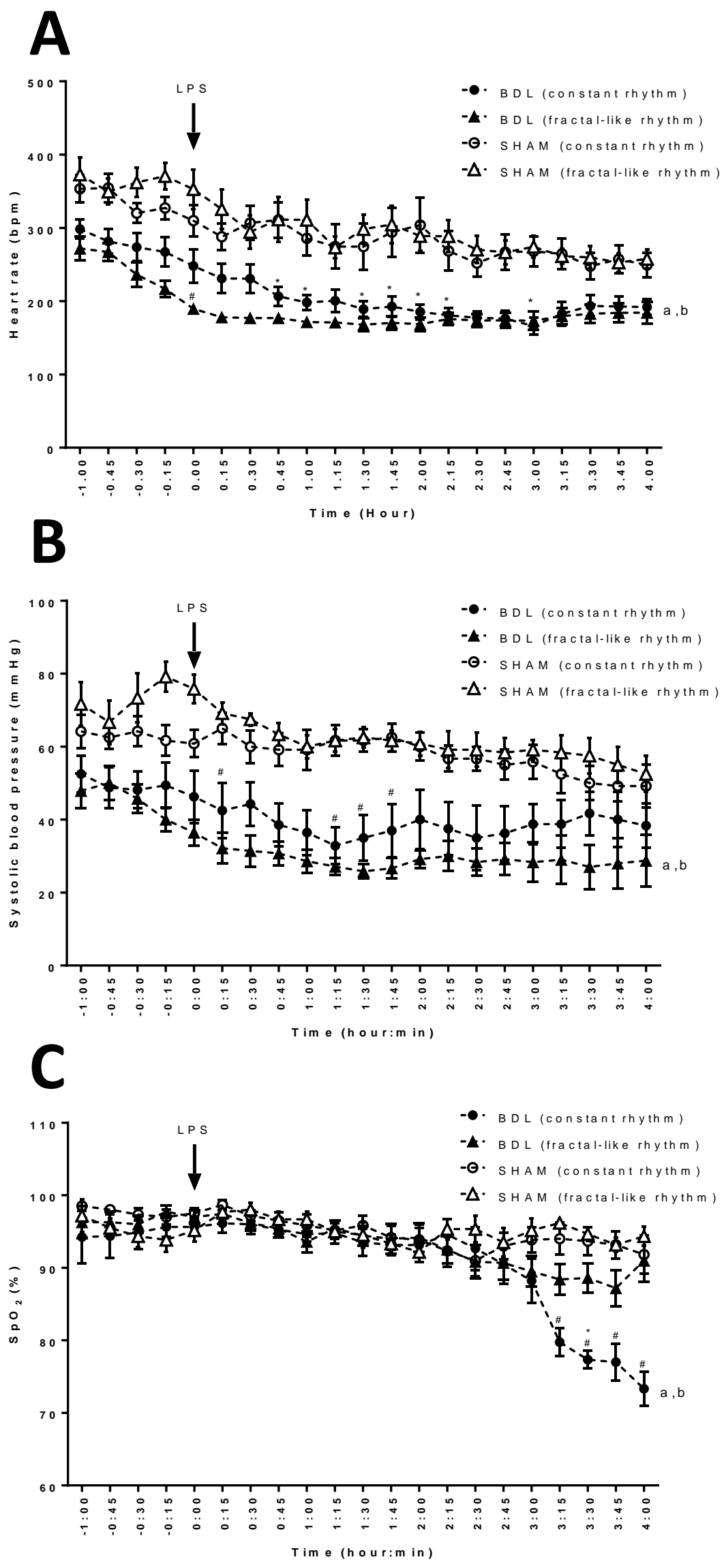


Figure 4. The effect of fractallike mechanical ventilation on total HRV (SDNN, A), shortterm HRV (SD1, B) and longterm HRV (SD2, C). a: $\mathrm{P}<0.05$ $B D L$ fractal vs. BDL constant. b: BDL constant ventilation vs. SHAM constant $\mathrm{P}<0.05$.

\section{A}
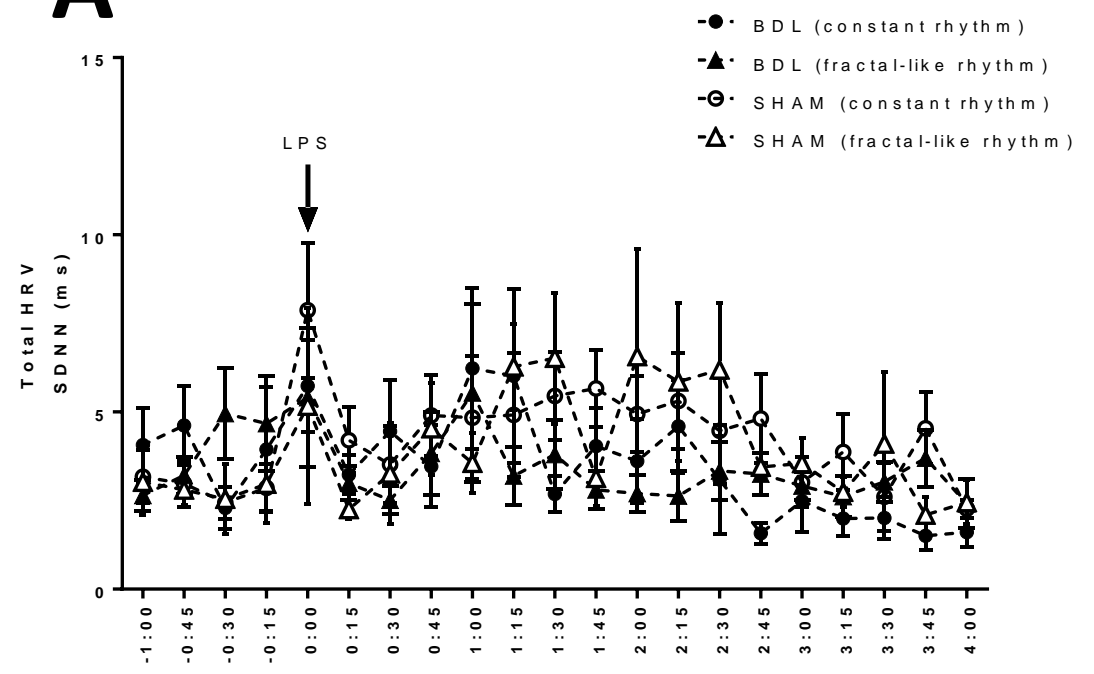

B

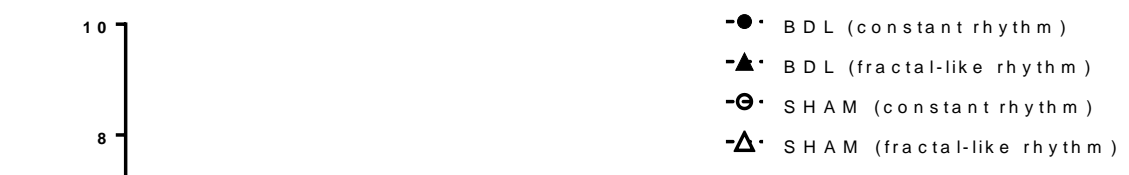

E

5
$\vdots$
$\vdots$
$\vdots$

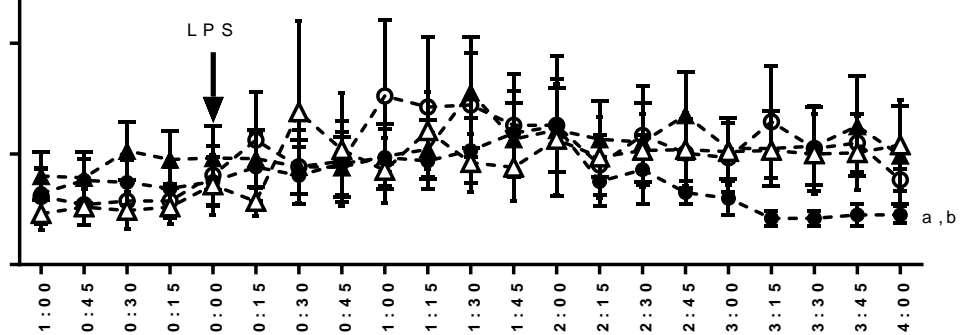

C
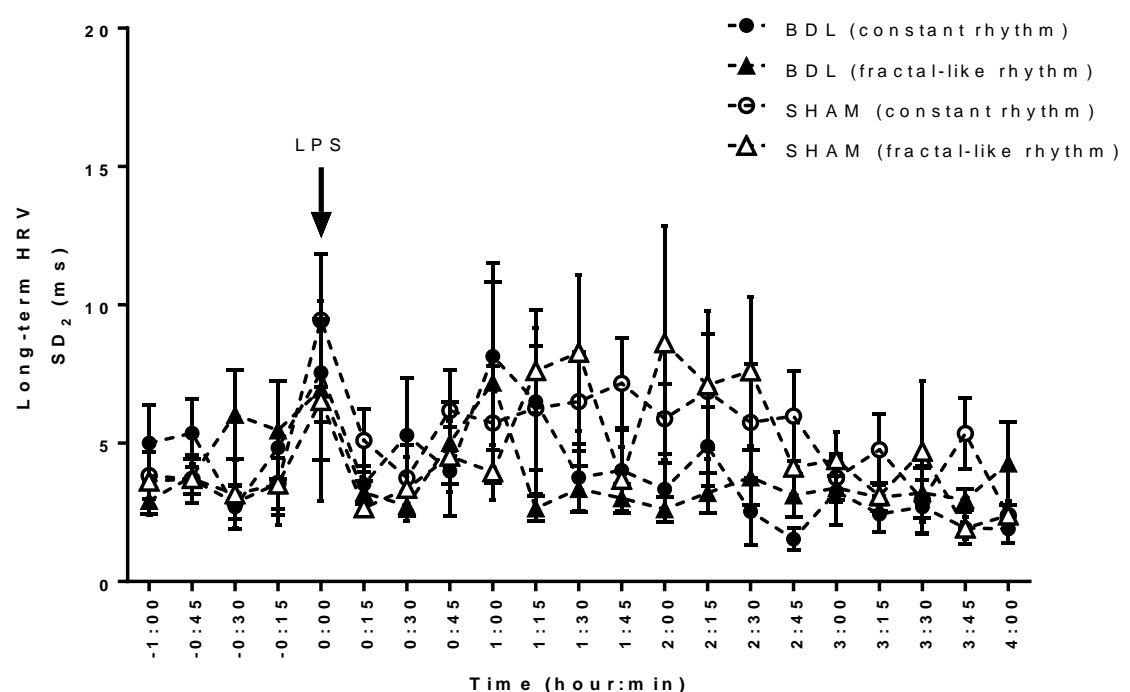
Figure 5. Kaplan-Meier survival curve in cirrhotic (BDL) or sham-operated (SHAM) control rats after acute endotoxin challenge. The rats were given either constant or fractal-like mechanical ventilation under general anaesthesia. A log-rank (Mantel-Cox) test showed a significant difference in mortality $(\mathrm{P}<0.05)$.

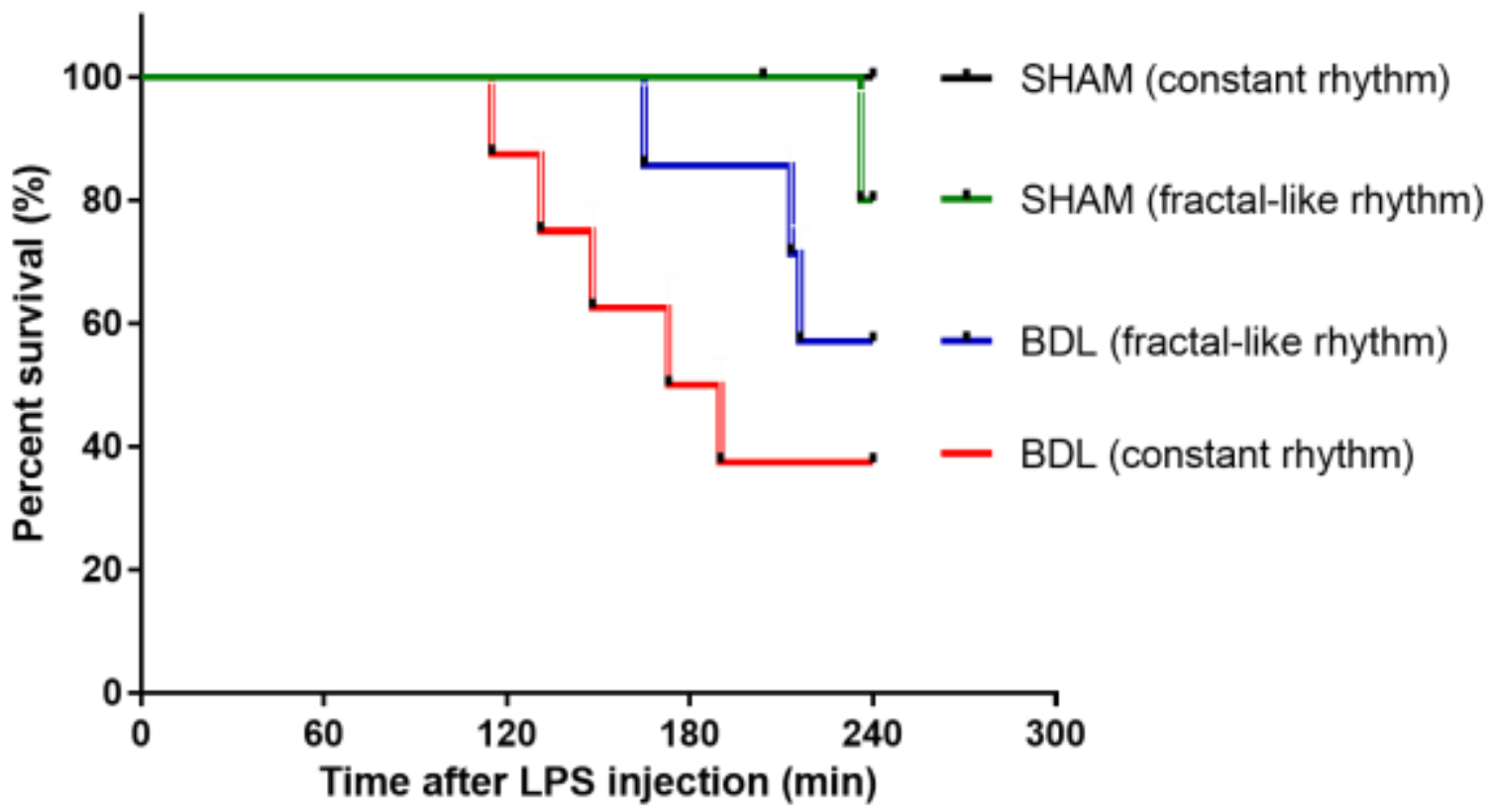

